UNIVERSIDADE FERERAL DO PARANÁ

VINICIUS VILLAS BOAS PETRONI

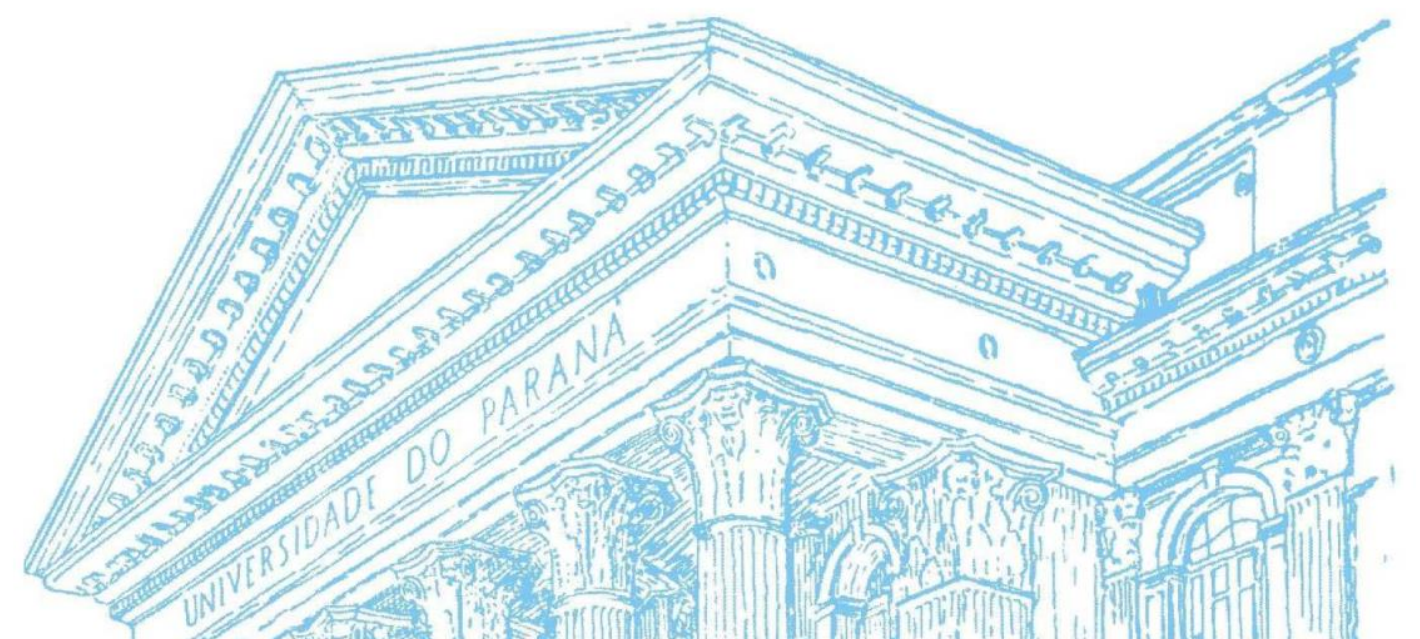

AVALIAÇÃO DA TRANSMISSÃO DE TEMPERATURA DA RESINA ACRÍLICA PARA OS OSSOS CORTICAL E MEDULAR POR MEIO DE

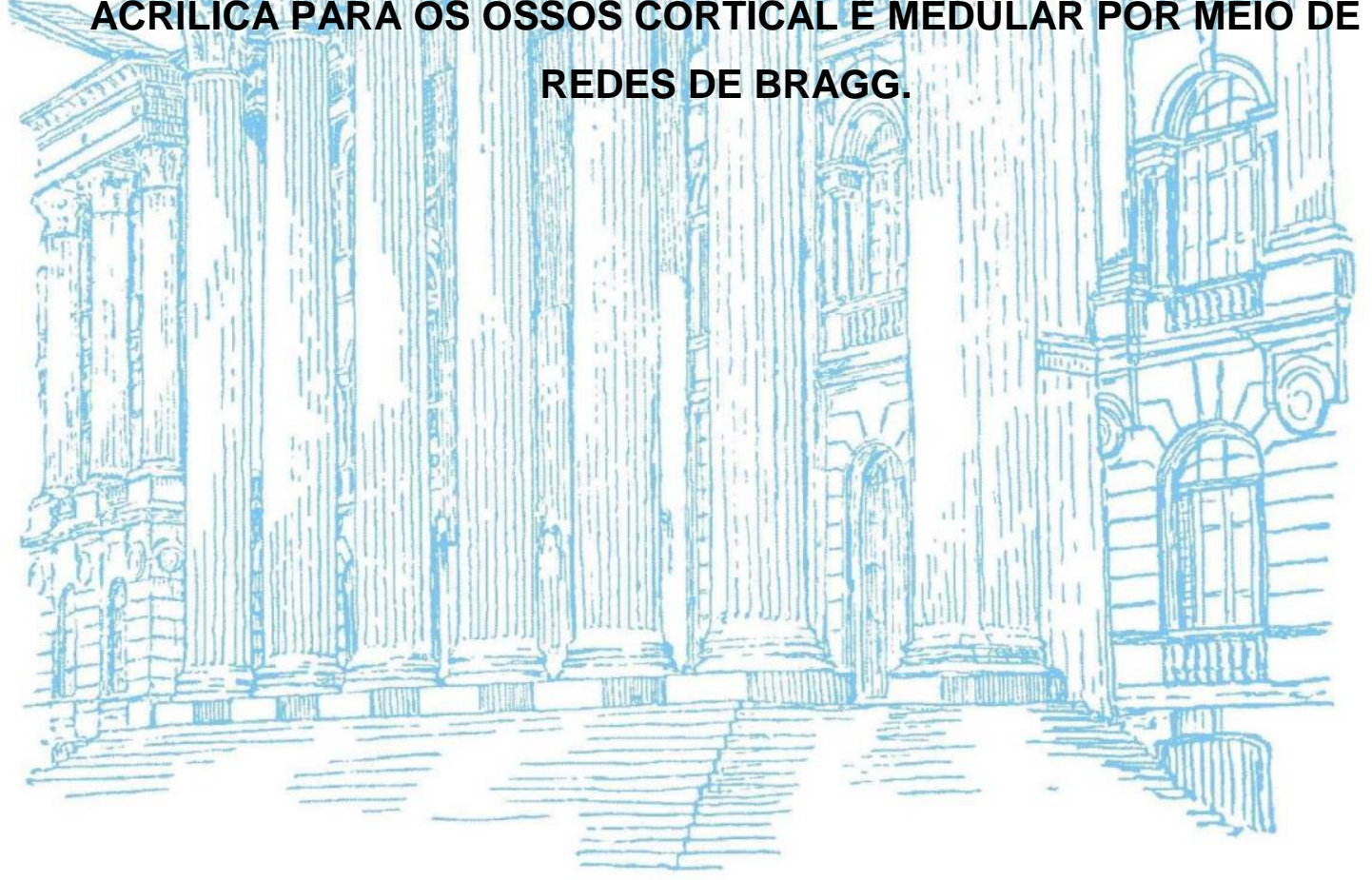

CURITIBA 


\title{
AVALIAÇÃO DA TRANSMISSÃO DE TEMPERATURA DA RESINA ACRÍLICA PARA OS OSSOS CORTICAL E MEDULAR POR MEIO DE REDES DE BRAGG.
}

\author{
Monografia apresentada como requisito \\ parcial à obtenção do título de Especialista, \\ Curso de Especialização em Prótese \\ Dentária, Setor de Ciências da Saúde, \\ Universidade Federal do Paraná. \\ Orientadores:
}

Orientador: Prof. Dr. Nerildo L. Ulbrich Coorientadora: Prof. Dra. Ana Paula Gebert de Oliveira Franco

CURITIBA 


\section{AGRADECIMENTOS.}

Agradeço, primeiramente, à minha família que sempre esteve presente e me apoiando em todos os momentos de minha vida.

À Ana Paula Gebert de Oliveira Franco, Pós-Doutoranda da UTFPR, pela orientação e esforço para confecção deste trabalho.

À equipe de profissionais do laboratório de ótica da UTFPR, que disponibilizaram seus equipamentos e estrutura para o desenvolvimento deste trabalho.

Aos amigos por facilitar o dia-a-dia de clínicas e aulas.

À namorada pelo companheirismo e ajuda nas diversas dificuldades. 


\section{RESUMO}

Objetivo: O objetivo do estudo foi avaliar a transmissão de temperatura da resina acrílica para os ossos cortical e medular por meio de Redes de Bragg. Também foi verificado se o sistema utilizado possui sensibilidade o suficiente para a análise, além de determinar se as alterações de temperatura podem causar necrose óssea. A partir do desenvolvimento desse estudo poderemos selecionar a resina com menor alteração de temperatura, evitando assim, qualquer tipo de alteração morfológica/dano irreversível que possa ocorrer à região óssea. Material e Métodos: Foram utilizados dois implantes Titamax $\mathrm{CM}$ com diâmetro de $4 \mathrm{~mm}$ e comprimento de $15 \mathrm{~mm}$. Estes foram instalados em um osso artificial da marca Sawbones que apresenta propriedades de módulo de elasticidade semelhantes aos ossos cortical e medular naturais. Sobre os implantes foram fixados os transfers de moldeira aberta, e então foi realizada a união dos mesmos com fio dental e resina acrílica utilizando-se da técnica de Nealon. As resinas selecionadas foram a Duralay e a GC Pattern LS. Duas perfurações perpendiculares aos implantes foram realizadas: a primeira na região cortical cervical, direcionada lateralmente ao corpo do implante; e a segunda na região apical medular, direcionada logo abaixo do corpo do implante. Um sensor foi inserido em cada uma das perfurações, e em seu entorno foi aplicada uma pasta térmica, que teve o objetivo de preencher a cavidade e transmitir o calor do implante diretamente para o sensor, tendo-se assim uma análise de variação de temperatura mais precisa. Os dados foram então transmitidos para um computador e analisados estatisticamente por meio de ANOVA 2 critérios e terste de múltiplas comparações de Games Howell $(\mathrm{p}<0.05)$. Resultados: Os resultados revelaram para Duralay cervical $0,99 \pm 0,33^{\circ} \mathrm{C}$, Duralay apical $0,82 \pm 0,37^{\circ} \mathrm{C}, \mathrm{GC}$ Pattern LS cervical $1,08 \pm 0,40^{\circ} \mathrm{C}$, GC Pattern LS apical $0,82 \pm 0,17^{\circ} \mathrm{C}$. Conclusão: Houve diferenças significativas entre os valores da Duralay apical e GC Pattern LS cervical, GC Pattern LS cervical e GC Pattern LS cervical. As redes de Bragg foram capazes de mensurar a transferência de temperatura do trasnferente e do implante para os ossos cortical e medular. Não há risco de necrose óssea pela utilização das resinas estudadas.

Palavras-chaves: Temperatura, Implante Dentário, Osso. 


\begin{abstract}
Objective: The purpose of this study was to evaluate the temperature transmission of the acrylic resin to the cortical and medullary bones by means of Bragg's gratting sensors. We will also check if the system used has enough sensitivity for the analysis, as well as determine if changes in temperature can cause bone necrosis. From the development of this study we can select the resin with the lowest temperature change, thus avoiding any type of morphological change / irreversible damage that may occur to the bone region. Material and Methods: Two Titamax CM implants with a diameter of $4 \mathrm{~mm}$ and a length of $15 \mathrm{~mm}$ were used. These were installed on an artificial bone of the Sawbones brand that exhibits modulus properties similar to natural cortical and medullary bones. The open tray transfers were fixed on the implants, and the dental floss and acrylic resin were joined using the Nealon technique. The resins Duralay and GC Pattern LS were selected. Two perforations perpendicular to the implants were performed: the first in the cervical cortical region, directed laterally to the implant body; And the second in the medullary apical region, directed just below the implant body. A sensor was inserted in each of the perforations, and in its surroundings a thermal paste was applied, that had the objective of filling the cavity and transmit the heat of the implant directly to the sensor, thus having a more precise analysis of variation of temperature. The data were then transmitted to a computer and then analyzed statistically. Results: The results revealed for cervical Duralay $0.99 \pm 0.33^{\circ} \mathrm{C}$, apical Duralay $0.82 \pm 0.37^{\circ} \mathrm{C}$, Cervical LS Pattern LS $1.08 \pm 0.40^{\circ} \mathrm{C}$, GC Pattern LS apical $0,82 \pm 0.17^{\circ} \mathrm{C}$. Conclusion: There were significant differences between the values of the Duralay apical and GC Pattern LS cervical, GC Pattern LS cervical and GC Pattern LS apical. The Bragg's gratting sensors were able to measure the transference of temperature from the transferent and the implant to the cortical and medullary bones. There is no risk of bone necrosis due to the use of the resins studied.
\end{abstract}

Key-Words: Temperature, Dental Implantation, Bone. 


\section{LISTA DE TABELAS}

TABELA 1: DESCRIÇÃO DAS RESINAS UTILIZADAS NO ESTUDO............ 18

TABELA 2: ESTATÍSTICA DESCRITIVA DE TEMPERATURA MÁXIMA DAS DIFERENTES RESINAS ACRÍLICAS TRANSFERIDAS PARA AS DIFERENTES REGIÕES DE ATUAÇÃO DOS SENSORES ...................... 20 


\section{LISTA DE FIGURAS}

GRÁFICO 1 - GRÁFICO DA ANÁLISE ESTATÍSTICA DA TEMPERATURA

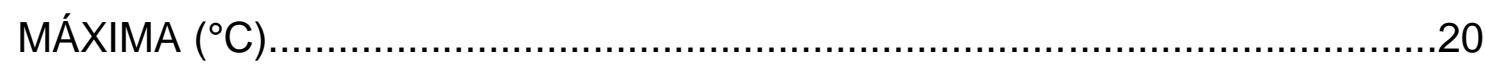

GRÁFICO 2 - CURVAS MÉDIAS DAS VARIAÇÕES DE TEMPERATURA DURALAY. .21

GRÁFICO 3 - CURVAS MÉDIAS DAS VARIAÇÕES DE TEMPERATURA

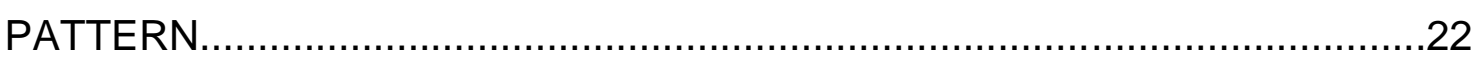




\section{SUMÁRIO}

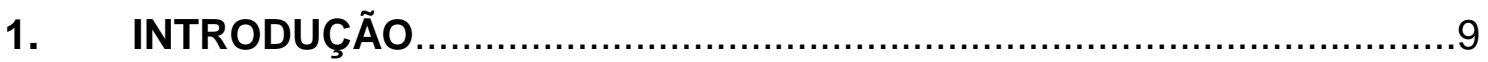

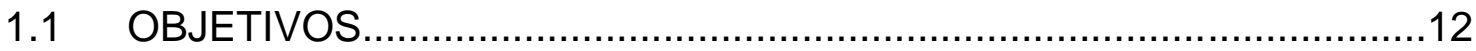

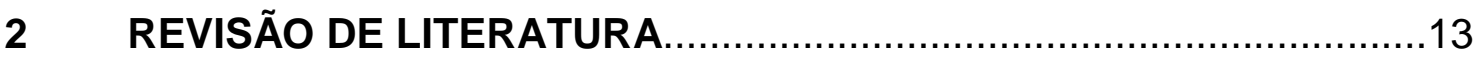

2.1 RELAÇÃO DA TEMPERATURA E ALTERAÇÕES ÓSSEAS.................13

2.2 RESINAS ACRÍLICAS E A ALTERAÇÃO DE TEMPERATURA.............15

3 MATERIAIS E MÉTODOS ........................................................... 17

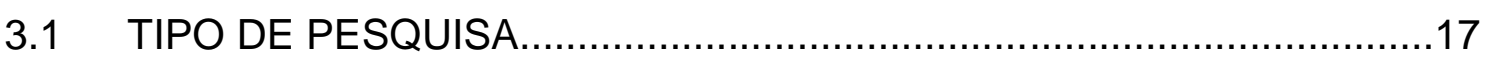

3.2 PROCEDIMENTOS E COLETAS DE DADOS .................................17

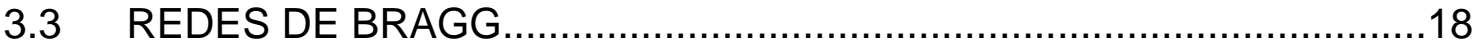

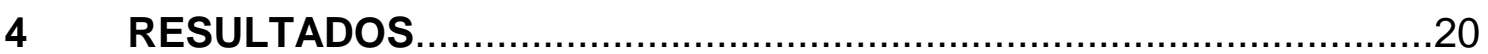

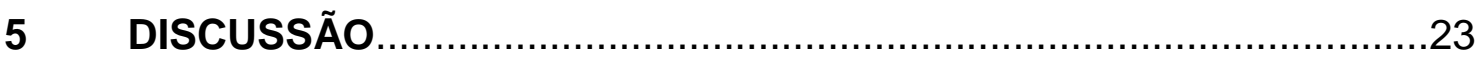

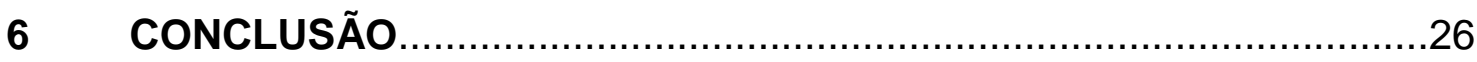

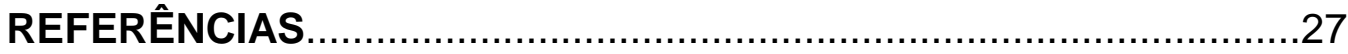




\section{INTRODUÇÃO}

Atualmente na Odontologia existem diversas maneiras de substituir um elemento dentário perdido, como o uso de próteses fixas convencionais ou próteses removíveis. Entretanto, nas últimas décadas, um método reabilitador vem ganhando grande destaque quanto à sua praticidade e bom resultado estético, os implantes dentários. Estes foram desenvolvidos pelo Professor PerIngvar Branemark, que descobriu em 1950 a ósseo-integração e, em seguida, em 1965, desenvolveu o primeiro implante dentário. O princípio básico da osseointegração consiste na formação óssea ao redor da superfície do implante (ALBREKTSSON; JOHANSSON, 2001). Para que isso ocorra de uma forma mais acelerada e eficiente, a superfície dos implantes recebe tratamentos químicos ou bioquímicos. Ambos levam a uma melhor ancoragem do implante ao osso (WENNERBERG; ALBREKTSSON, 2010). Esse fato possibilitou que reabilitações protéticas sobre implante mais extensas pudessem ser realizadas, trazendo uma melhor qualidade de vida aos pacientes. Como visto em estudo realizado por Rieko et al.(2012), pacientes com experiência anterior de uso de próteses parciais removíveis e totais, quando reabilitados com o uso de implantes, tiveram uma melhora na função e se sentiram mais confortáveis com a estabilização dos aparatos protéticos.

É essencial que o encaixe da prótese sobre o implante se dê de forma passiva para que nenhum tipo de força deletéria atue sobre o mesmo. (GUICHET et al. 2000) Portanto, o clínico deve realizar uma moldagem fiel, e que demonstre o posicionamento tridimensional exato do implante na cavidade bucal. Para isso existem diferentes técnicas de moldagem com esse objetivo, sendo que neste artigo iremos focar na técnica de moldagem aberta. Esta técnica é utilizada no caso de múltiplos implantes e consiste em unir os transferentes com fio dental e resina acrílica previamente à moldagem em si. (ASSIF et al. 1999). Desta maneira, diminui-se a probabilidade de ocorrer qualquer tipo de rotação dos transferentes no material de impressão. Existe uma pequena variação da técnica utilizada por alguns autores (VIGOLO et al. 2004) onde a resina acrílica é seccionada e então unida novamente antes da moldagem. Outros autores (CABRAL; GUEDES, 2007) sugeriram uma espera 
de 17 minutos na polimerização da resina antes do seccionamento, para evitar a contração da resina e qualquer tipo de alteração que isso venha a trazer.

As resinas acrílicas utilizadas neste estudo são a Duralay e a GC Pattern LS. A Duralay é uma resina do tipo Metacrilato, composta por um copolímero de metacrilato plastificável. A resina GC Pattern LS também é um metacrilato, mas composta principalmente por polimetilmetacrilato. Ambas as resinas, durante a sua polimerização, sofrem exotermia e contração. Mas como descrito no site da resina GC Pattern LS, os fabricantes garantem que isso ocorre em menor grau no seu produto (www.gcamerica.com/lab/products/PATTERN_RESIN_LS). A regeneração tecidual óssea que deve ocorrer após o preparo do osso e posicionamento do implante está diretamente relacionada com a vascularização desse tecido, onde a adição de um trauma térmico pode ser suficiente para retardar ou impedir a mesma. (ERIKSSON, ALBREKTSSON, 1983) Dependendo da intensidade térmica gerada pode ocorrer até mesmo a indução de necrose óssea. Lundskog (1972) descreve em sua pesquisa que a temperatura de $50^{\circ} \mathrm{C}$, aplicada durante 30 segundos na superfície óssea é suficiente para causar necrose óssea. Para se obter a osseointegração e evitar os efeitos nocivos ao tecido ósseo, a manutenção da temperatura do osso abaixo de $47^{\circ} \mathrm{C}$ é essencial. (ERIKSSON, ALBREKTSSON, 1983),

As Redes de Bragg são sensores de fibra ótica sensíveis às variações de temperatura, tensão, pressão, deformações, umidade e doses de radiação. Esses sensores de fibra ótica têm sido utilizados amplamente em diversos setores, porém atualmente vêm sendo aplicados em análises biomédicas (BABCHENKO, 1999; PULIDO, 2016; OTTEVAERE, 2005; FRESVIG, 2008; MIHAILOV, 2012; KARAM, 2016; FRANCO, 2015), pois possuem algumas vantagens em comparação aos sistemas convencionais, como por exemplo, seu tamanho reduzido, alta sensibilidade à variação de temperatura e contração, imunidade à interferência eletromagnética entre outros. (TALAIA et al., 2007) (CARVALHO et al., 2011)

Estão presentes na literatura estudos que avaliam a transmissão de calor das resinas acrílicas utilizadas na união dos transferentes para os ossos 
cortical e medular por meio dos métodos de termopar (ERIKSSON, 1982; ORMIANER, 2000), porém não existem estudos que avaliem essa condição por meio de sensores de fibra ótica.

Através de artigos relacionados, pode-se afirmar que as resinas acrílicas utilizadas para a união de transferentes não chegam a causar necrose óssea, mas alterações teciduais localizadas. Então, a partir deste estudo, teremos a possibilidade de selecionar a resina que venha a trazer a menor alteração térmica, e, por consequência, evitar ou amenizar qualquer tipo de alteração morfológica ou dano irreversível que possa a ocorrer à região óssea. 


\subsection{OBJETIVOS}

- Objetivo Geral

Avaliar a alteração de temperatura nos ossos cortical e medular gerados pela resina acrílica na união dos transferentes.

- Objetivos Específicos

- Comparar a temperatura exotérmica das duas resinas (GC Pattern LS e Duralay)

- Comparar a alteração de temperatura nos ossos cortical e medular.

- Verificar se o sistema apresenta sensibilidade suficiente para a análise.

- Determinar se as alterações de temperatura podem causar necrose óssea. 


\section{REVISÃO DE LITERATURA.}

\subsection{RELAÇÕES DA TEMPERATURA E ALTERAÇÕES ÓSSEAS}

Em estudo realizado por Eriksson e Albrektsson (1982), cinco lebres foram submetidas à instalação de implantes em sua tíbia para posterior aquecimento do mesmo a $53^{\circ} \mathrm{C}$ por um minuto.

As alterações encontradas foram as seguintes:

- Circulação sanguínea parou ao atingir a temperatura. Depois retornou ao normal. Uma semana depois, nenhum dos vasos estava mais presente. Novos vasos estavam se formando.

- Células gordurosas diminuíram em número. Depois retornaram à situação normal.

- Houve grande reabsorção óssea, com pouca ou nenhuma recuperação ao longo das semanas.

Em outro estudo realizado por Erikkson e Albrektsson (1983), 15 coelhos, machos e fêmeas foram sedados (anestesia geral) e então submetidos à instalação de um implante em sua tíbia. Esse implante era oco na região cervical, com dois cilindros de vidro separados por espaços de $100 \mathrm{~nm}$. Então se esperava a cicatrização óssea depois de oito semanas, onde o implante era aquecido e depois analisado. Os coelhos foram separados em três grupos com 5 coelhos em cada grupo: Grupo A (Temperatura: 50ํㅡ, Tempo: 1 minuto); Grupo B (Temperatura $47^{\circ} \mathrm{C}$, Tempo: 5 minutos); Grupo C (Temperatura: $47^{\circ} \mathrm{C}$, Tempo: 1 minuto). Após o aquecimento do osso, o mesmo foi analisado em intervalos de tempo regularmente espaçados. Os resultados foram separados em: Efeitos agudos e a longo prazo.

- Resultados do grupo A:

- Efeitos agudos: Hiperemia, dilatação venular e arteriolar quando a temperatura foi elevada de 40 a $41^{\circ} \mathrm{C}$. Ao chegar aos $50^{\circ} \mathrm{C}$, o fluxo sanguíneo parou em alguns dos vasos 
menores. Uma hora após, a microcirculação se recuperou um pouco.

- Efeito a longo prazo: Pequeno aumento do fluxo sanguíneo, os vasos com fluxo parado haviam desaparecido e não era mais observada hiperemia. Células gordurosas foram reabsorvidas e tiveram alteração em sua cor. O tecido ósseo sofreu alterações a partir da terceira semana pósaquecimento, onde se presenciou que $30 \%$ do osso da região foi reabsorvido e substituído por células gordurosas.

- Resultados do Grupo B:

- Efeitos agudos: Arteríolas e vênulas sofreram dilatação, resultando em diâmetro vascular aumentado.

- Efeitos a longo prazo: Diâmetro dos vasos levemente aumentado, e depois de 4 a 5 dias se normalizaram. Ocorreu reabsorção das células gordurosas. Em quatro animais, ocorreu reabsorção óssea em aproximadamente 20 a $30 \%$ a região óssea original. Concomitante à essa reabsorção, ocorria uma invasão de células gordurosas na região.

- Resultados do Grupo C:

- Efeitos agudos: Resposta vascular análoga àquela observada no grupo B.

- Efeitos a longo prazo: Reabsorção de células gordurosas foi observada em todos os animais, porém de forma mais lenta. A reabsorção óssea foi observada em apenas dois animais, e ocorreu em apenas $10 \%$ da região óssea original.

Este estudo indicou que o tecido ósseo é sensível ao aquecimento a $47^{\circ} \mathrm{C}$ e a traumas cirúrgicos intensos, recomendando um controle cirúrgico adequado, facilitando a recuperação óssea. 


\subsection{RESINAS ACRÍLICAS E A ALTERAÇÃO DE TEMPERATURA.}

Vallittu (1996) analisou o pico das temperaturas de resinas acrílicas polimerizadas em diferentes volumes e em diferentes temperaturas iniciais. Ele utilizou as seguintes resinas: Temp plus, Dentalon Plus, Palavit G, Palapress, Pro Base Hot e Pro Base Cold. As quantidades de material variaram a partir de $750 \mathrm{~mm}^{3}$ a $22^{\circ} \mathrm{C}$ até $3000 \mathrm{~mm}^{3}$ a $37^{\circ} \mathrm{C}$ sendo medidas com termopares. Notouse que quanto maior a quantidade de resina utilizada, maior seria a temperatura atingida. $\mathrm{O}$ maior pico de temperatura encontrado foi referente à resina Pro Base Hot, chegando a $101^{\circ} \mathrm{C}$ quando aplicada em quantidades de $3000 \mathrm{~mm}^{3}$. A partir dos dados analisados, pode-se perceber que a variação de temperatura das resinas pode ser muito elevada, correndo riscos de alterações pulpares e de superfícies de suporte dentais.

Ormianer et al. (2000) realizaram um estudo onde avaliou a alteração de temperatura durante a autopolimerização de resinas acrílicas aplicadas diretamente sobre o abutment do implante. Um implante de titânio de $10 \mathrm{~mm}$ de comprimento, $4 \mathrm{~mm}$ de diâmetro e uma cinta metálica de $2 \mathrm{~mm}$ foi instalado em uma mandíbula de resina imersa em água, com 3 termopares acoplados (1 apical, 1 cervical e outro diretamente na água). Duas marcas de resina foram aplicadas diretamente no abutment (Duralay e GC Pattern LS) através da técnica de Nealon e da técnica da bolinha em dois tipos diferentes de abutments: cinta pequena (12,3 $\mathrm{mm}$ de comprimento e $8.9 \mathrm{~mm}$ de diâmetro) e média (12,3 mm de comprimento e 11.0 mm de diâmetro).

Nos resultados, notam-se alguns fatos:

- A alteração de temperatura foi maior nas cintas médias quando comparadas em ambas as técnicas, exceto com a resina Duralay durante a técnica da bolinha.

- Para as cintas de mesmo tamanho, a diferença de técnica não foi significativa, exceto quando a resina GC Pattern LS foi aplicada na banda média.

- As alterações apicais foram menores que as cervicais, sendo de no máximo $2^{\circ} \mathrm{C}$ para a primeira e de $6^{\circ} \mathrm{C}$ na segunda. 
Sendo assim, o uso descontrolado de grandes quantidades de resina acrílica na união de postes de moldagem ou diretamente aplicada aos abutments pode transmitir significantes níveis de energia térmica. Dependendo de sua variação e duração, pode ocasionar alterações teciduais, devendo ser cuidadosa a sua aplicação.

Kazemi et al. (2012) realizou um estudo onde analisou resinas acrílicas aplicadas diretamente ao abutment, usando-se de termopares para medição da alteração de temperatura. 20 implantes (10 com diâmetro 3.3 e $10 \mathrm{com}$ diâmetro de 4.1) foram instalados em osso fresco extraído do ilíaco de carneiros (sacrificados 2 horas antes da instalação dos implantes). Foi utilizado um guia metálico parafusado ao implante para guiar as perfurações e colocação dos termopares. Três resinas acrílicas foram utilizadas: Duralay, Tempron e Acropars. Pó e líquido foram misturados e colocados em um molde com formato de um molar, limitando a quantidade utilizada de resina semelhante à confecção clínica de coroas provisórias. Este molde foi então posicionado no abutment, removendo-se os excessos logo em seguida.

Os resultados encontrados demonstraram que a Duralay apresentou a maior temperatura $\left(48.2^{\circ} \mathrm{C}\right)$, enquanto que a Acropars apresentou a menor $\left(45,2^{\circ} \mathrm{C}\right)$. As três resinas tiveram uma diferença significativa entre elas, mas $\mathrm{O}$ tamanho das plataformas não teve influência sobre as temperaturas. A transmissão de calor para o osso foi de $1^{\circ} \mathrm{C}$ na região cervical e de $0,5^{\circ} \mathrm{C}$ na região apical.

Os estudos que relacionam o uso de resinas acrílicas e sua exotermia com a injúria de tecidos de sustentação são escassos. 


\section{MATERIAIS E MÉTODOS.}

\subsection{TIPO DE PESQUISA}

O presente estudo se enquadra aos fins que se destina como pesquisa experimental.

\subsection{PROCEDIMENTOS E COLETAS DE DADOS}

Foram selecionados dois implantes cone Morse da marca Titamax, com $4 \mathrm{~mm}$ de diâmetro e $15 \mathrm{~mm}$ de comprimento.

O osso artificial utilizado foi da marca Sawbones, que apresenta propriedades de módulo de elasticidade semelhantes aos ossos cortical e medular naturais. Nele foram demarcadas, com o auxílio de um modelo, as distâncias entre os implantes, simulando a necessidade de uma prótese sobre múltiplos implantes. A localização dos pilares de implante foi referente aos dentes 44 e 46, com ausência do dente 45. Os implantes foram inseridos no osso artificial com perfurações escalonadas com brocas indicadas pelo fabricante, na velocidade de $600 \mathrm{rpm}$ em contra-ângulo redutor de velocidade, na proporção de 20:1. Em seguida, também com o auxílio do contra-ângulo, em velocidade reduzida $(250 \mathrm{rpm})$, com $5 \mathrm{~N}$ de torque final, eles foram aprofundados, ficando $1 \mathrm{~mm}$ abaixo da borda de perfuração.

Sobre esses mesmos implantes foram instalados os postes de moldagem para a técnica aberta, sendo que estes foram posteriormente unidos com fio dental e resina acrílica.

As resinas selecionadas para o presente estudo foram a Duralay e a GC Pattern LS. Suas composições estão especificadas na Tabela 1.

As resinas acrílicas foram aplicadas com um pincel de pelo de Marta fino, utilizando-se da técnica de Nealon, onde se molha o pincel no monômero e em seguida no copolímero da resina acrílica, para então ser levado em contato com o fio dental. O pó e o líquido das resinas acrílicas foram pesados em uma balança analítica de precisão AUW220D (Shimadzu, North America) para padronização da quantidade $(0,14 \mathrm{~g}$ de pó e $0,05 \mathrm{~g}$ de líquido) de resinas acrílicas utilizadas em cada esplintagem. Foi também padronizada a 
quantidade de dez pinceladas, sendo três para cada poste de moldagem e 4 sobre o fio dental entre os postes de moldagem. Foram posicionados dois sensores de fibra óptica, um próximo à região cervical, posicionado no osso cortical, e o outro próximo à região apical, posicionado no osso medular. Estavam posicionados perpendicularmente ao implante, circundados por uma pasta térmica (Implastec Eletroquímica), com o objetivo de transmitir o calor do implante para o sensor de fibra ótica. Os experimentos foram realizados em um ambiente de temperatura controlada de $21^{\circ} \mathrm{C}$ e umidade também controlada.

Tabela 1: Descrição das Resinas utilizadas no estudo.

\begin{tabular}{|c|c|c|c|}
\hline $\begin{array}{l}\text { Nome } \\
\text { do } \\
\text { Produto }\end{array}$ & $\begin{array}{l}\text { Tipo de } \\
\text { Resina }\end{array}$ & Composição & Fabricante \\
\hline Duralay & Metacrilato & $\begin{array}{l}\text { Copolímero de metacrilato } \\
\text { plastificável, Monômero de } \\
\text { metilmetacrilato, Parafina, } \\
\text { Óleo Mineral. }\end{array}$ & $\begin{array}{ll}\text { Reliance Dental } & \text { MFG } \\
\text { Company, Illinois, EUA } & \end{array}$ \\
\hline Pattern & Metacrilato & $\begin{array}{l}\text { Polimetilmetacrilato, } \\
\text { Polietilmetacrilato, peróxido } \\
\text { de benzoila. }\end{array}$ & GC America, Illinois, EUA \\
\hline
\end{tabular}

\subsection{REDES DE BRAGG}

As redes de Bragg foram gravadas em fibra ótica convencional (Draktel, ESMF) não hidrogenada, utilizando iluminação sob máscara de fase no comprimento de onda de $193 \mathrm{~nm}$ (laser excímero ArF). A amplitude das redes foi padronizada em $11 \mathrm{~dB}$ acima do patamar de ruído. Todas as redes foram produzidas nas mesmas condições, nos laboratórios do Núcleo de Dispositivos Fotorrefrativos da UTFPR. O setup ótico foi montado com a utilização de um acoplador ótico, para que fosse possível a utilização de sensores em comprimentos de onda diferentes simultaneamente. Um interrogador de redes de Bragg Mícron Optics SM125 foi utilizado para medição dos espectros de reflexão, cujo deslocamento foram determinados os valores de contração e 
então todos os dados foram enviados e salvos com a utilização do software disponibilizado pelo fabricante. 


\section{RESULTADOS}

O teste ANOVA dois critérios e teste de múltiplas comparações de Games Howell revelaram que as variações de temperatura máxima apresentaram valores significativamente diferentes entre Duralay apical e GC Pattern LS cervical ( $p=0.020$ ), GC Pattern LS cervical e GC Pattern LS apical $(p=0.021)($ Tabela 2$)$.

Tabela 2. Estatística descritiva de temperatura máxima das diferentes resinas acrílicas transferidas para as diferentes regiões de atuação dos sensores.

\begin{tabular}{|c|c|c|c|}
\hline Resina & $\begin{array}{c}\text { Região de } \\
\text { atuaçãica } \\
\text { dos } \\
\text { Sensores }\end{array}$ & Média $\left({ }^{\circ} \mathbf{C}\right)$ & $\begin{array}{c}\text { Desvio } \\
\text { Padrão }\end{array}$ \\
\hline Duralay & Cervicalabc $^{\text {abc }}$ & 0,99 & 0,33 \\
\cline { 2 - 4 } & Apical $^{\text {ab }}$ & 0,82 & 0,37 \\
\hline \multirow{2}{*}{$\begin{array}{c}\text { GC Pattern } \\
\text { LS }\end{array}$} & Cervical $^{\text {ac }}$ & 1,08 & 0.40 \\
\cline { 2 - 4 } & Apical $^{\mathrm{ab}}$ & 0,82 & 0.17 \\
\hline
\end{tabular}

*Letras minúsculas diferentes revelam diferenças significativas na coluna $(\mathrm{p}<$ $0,05)$.

Gráfico 1. Gráfico da análise estatística da temperatura máxima $\left({ }^{\circ} \mathrm{C}\right)$ das diferentes resinas acrílicas utilizadas nas diferentes regiões de atuação dos sensores.

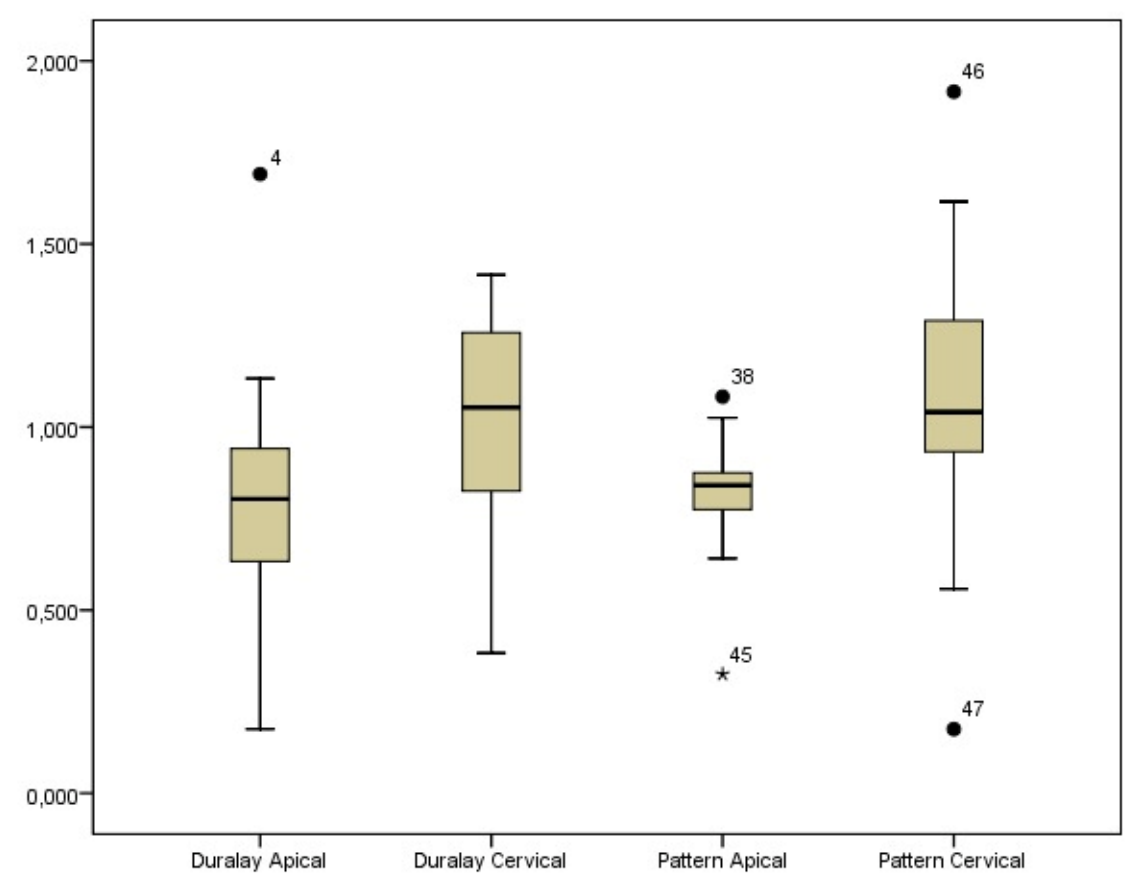


No gráfico 2 pode-se observar a curva média da variação de temperatura para a resina acrílica Duralay nas regiões cervical e apical ao implante. O início da elevação da temperatura para as duas regiões ocorreu no tempo de 4,16 min, atingindo seu pico em 21,66 min, a partir do qual a curva começa a decrescer, havendo uma tendência de estabilização próxima ao tempo de 58,33 min.

Gráfico 2. Curvas médias das variações de temperatura para a resina acrílica Duralay nas regiões cervical e apical ao implante.

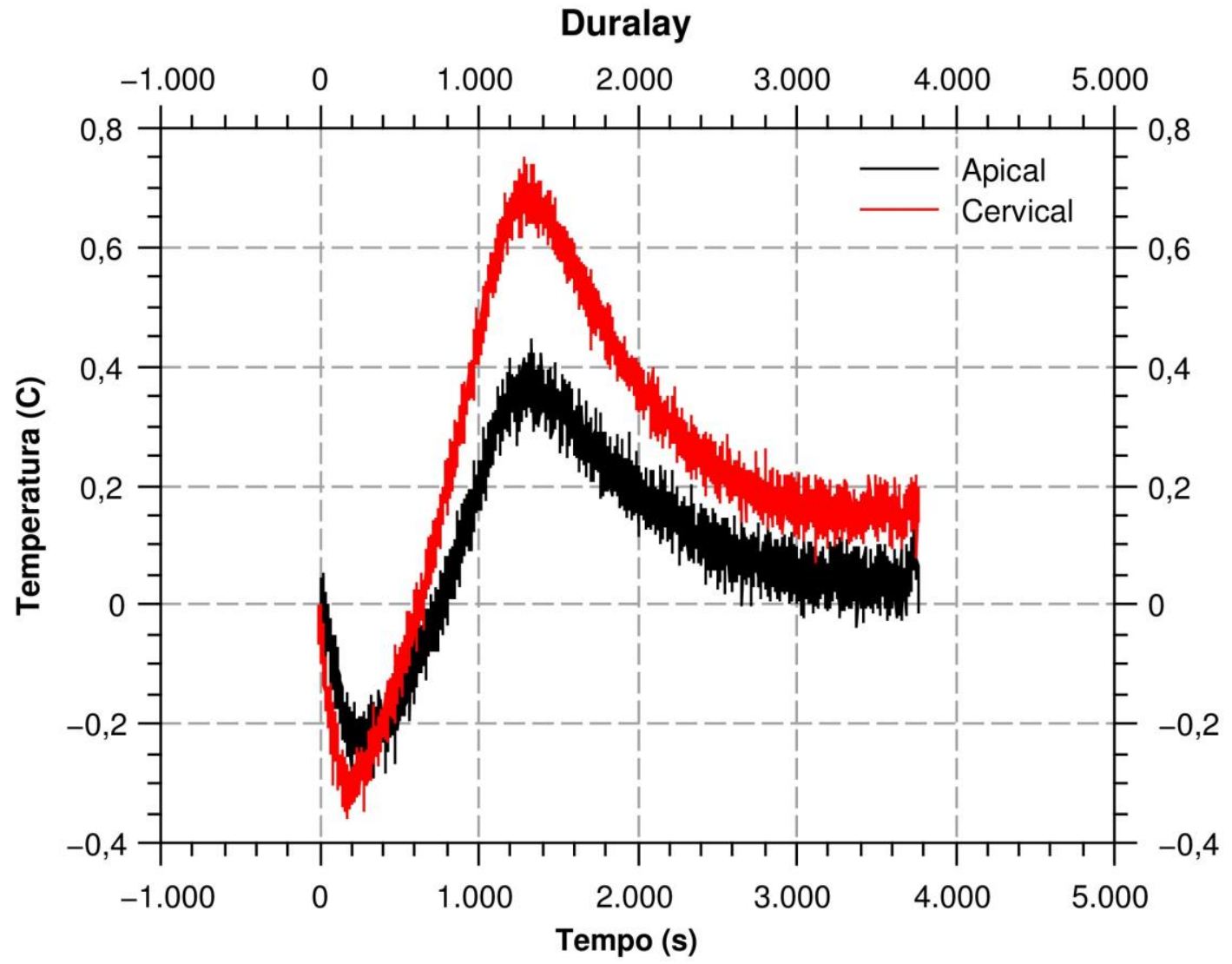

No gráfico 3 observa-se a curva média da variação de temperatura para a resina acrílica GC Pattern LS nas regiões cervical e apical ao implante. O início da elevação da temperatura para as duas regiões ocorreu no tempo de 1,66 min, atingindo seu pico em 9,16 min, a partir do qual a curva começa a decrescer, havendo uma tendência de estabilização próxima ao tempo de 50 $\min$. 
Gráfico 3. Curvas médias das variações de temperatura para a resina acrílica GC Pattern LS nas regiões cervical e apical ao implante.

\section{Pattern}

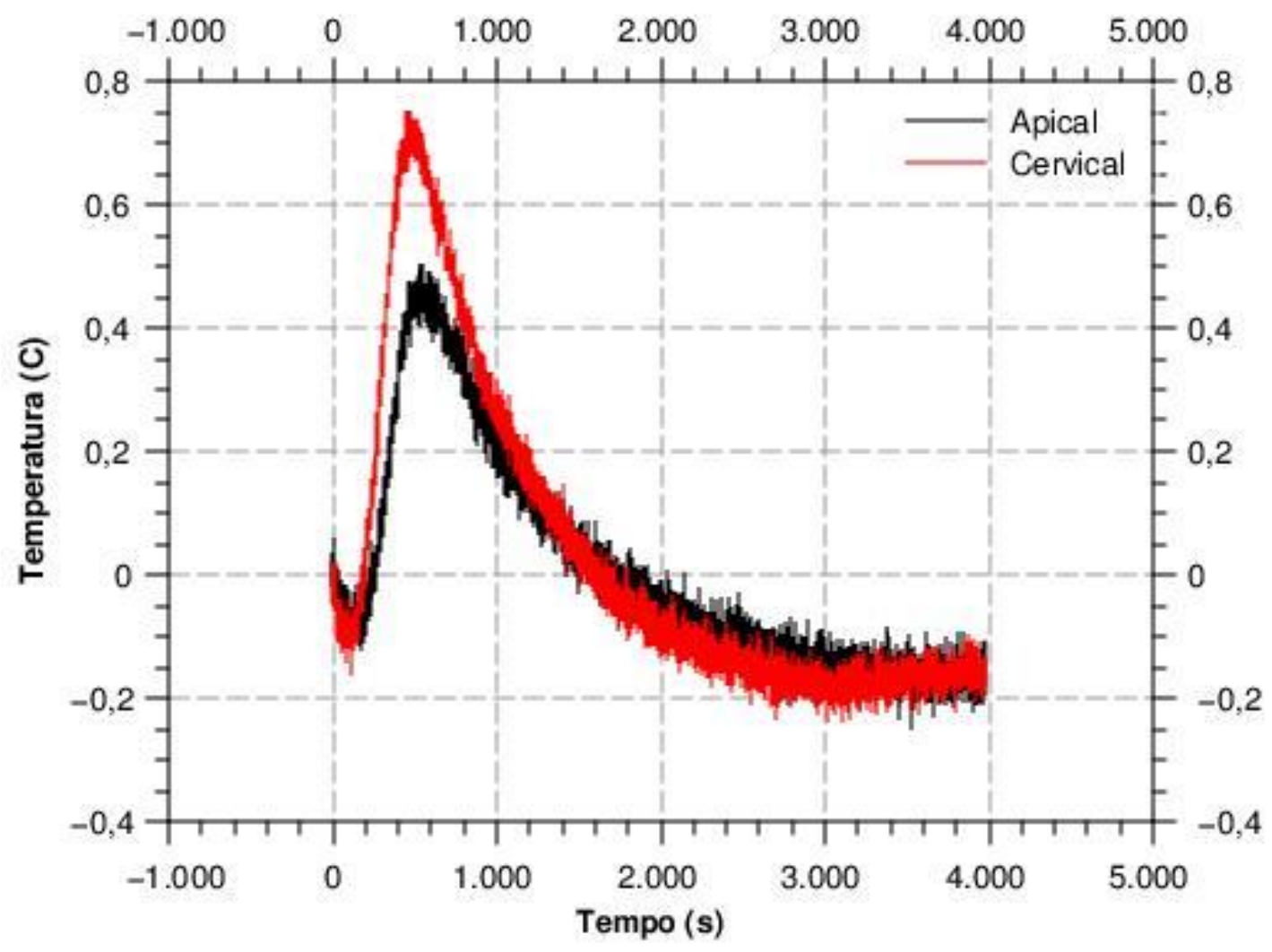




\section{DISCUSSÃO}

Os materiais poliméricos apresentam reação exotérmica durante a polimerização. Estudos anteriores revelaram uma elevação média de temperatura máxima de $16,24^{\circ} \mathrm{C}$ para a GC Pattern LS e de $43,43^{\circ} \mathrm{C}$ para a Duralay (FATIGA et al., 2015). O presente estudo visa identificar a transferência de calor da resina acrílica através do poste de moldagem e do implante até os ossos cortical e medular. A literatura mostra que temperaturas elevadas podem causar necrose óssea e, consequentemente, prejudicar o processo de osseointegração dos implantes. Como visto em estudo conduzido por Albrektsson ${ }^{25}$, a temperatura necessária para causar danos ao osso é de $47^{\circ} \mathrm{C}$ aplicada durante um minuto, onde se observou reabsorção óssea mínima nos casos encontrados e substituição do tecido ósseo perdido por células gordurosas. No presente estudo, pudemos presenciar que a alteração máxima de temperatura ocorreu com a resina GC Pattern LS $\left(1,08 \pm 0,40^{\circ} \mathrm{C}\right)$ cervical. Porém, este estudo demonstrou que a temperatura mais elevada encontrada nas superfícies apical e cervical dos implantes, ainda está abaixo dos limites de temperatura associados à alterações ósseas.

No presente estudo, a média de temperatura encontrada na região cervical dos implantes foi de $1,08^{\circ} \mathrm{C}$ para a resina GC Pattern LS e $0,99^{\circ} \mathrm{C}$ para a resina Duralay, não corroborando com estudo realizado por Ormianer et al (2000) onde a variação de temperatura encontrada na região cervical foi de $4^{\circ} \mathrm{C}$ a $5^{\circ} \mathrm{C}$. Essa inconsistência pode se dar devido ao fato da quantidade de resina acrílica aplicada e sua localização. Ormianer aplicou uma quantidade intermediária de resina diretamente sobre o implante diferentemente do presente estudo, onde uma quantidade mínima de resina foi aplicada por sobre o fio dental que unia os trasferentes instalados sobre a plataforma do implante.

Já no estudo de Kazemi et al (2012), a média de temperatura mais elevada para a região cervical foi da resina Duralay $\operatorname{com} 1,04^{\circ} \mathrm{C}$, e para a região apical, a média de temperatura encontrada foi de $0.5^{\circ} \mathrm{C}$, também com a resina Duralay. Os resultados obtidos neste estudo corroboram com aqueles encontrados por Kazemi, onde a média de temperatura encontrada na região cervical, com relação à resina Duralay, foi de $0,99^{\circ} \mathrm{C}$, enquanto que a média de 
temperatura apical foi de $0,82^{\circ} \mathrm{C}$. Leva-se a crer que a proximidade de valores se deu devido à padronização da quantidade aplicada, se aproximando daquelas encontradas na prática diária.

As curvas das duas resinas nas duas posições revelaram nos primeiros minutos (de 0 a 4,16 min para a Duralay; de 0 a 1,66 min para a GC Pattern LS) uma redução dos valores médios de temperatura sugestivos da ocorrência de uma contração das resinas entre os postes de moldagem, o que reflete em deformação nos ossos cortical e medular nas regiões cervical e apical ao implante.

Observou-se que a resina acrílica GC Pattern LS apresentou um aumento da temperatura em menor tempo mostrando que sua reação exotérmica ocorre de forma mais rápida se comparada à da Duralay. Apesar de o fabricante da GC Pattern LS relatar menores valores de temperatura se comparada à Duralay, pode-se observar pelos resultados do presente estudo que houve diferenças estatísticas significativas dos valores máximos de temperatura entre GC Pattern LS cervical e GC Pattern LS apical, e Duralay apical e GC Pattern LS cervical.

As duas resinas acrílicas demonstraram estabilização da temperatura ao final de 1 hora, demonstrando que o tempo utilizado no estudo permite avaliar de forma integral e suficiente a reação exotérmica.

O método proposto foi realizado de acordo com a utilização clínica das resinas acrílicas para união dos postes de moldagem por meio da técnica de Nealon, porém procurou-se padronizar a quantidade de pó e líquido na pesagem em balança analítica e utilização de 10 pinceladas visando obter resultados passíveis de reprodutibilidade.

As redes de Bragg são sensores de fibra ótica da engenharia que têm sido aplicados nas áreas biomédicas pelo fato de poderem ser aplicados in situ, ou seja, em regiões de pequenas dimensões exatamente no local da utilização clínica dos materiais se for considerada a área odontológica. São exemplos disso estudos in vitro em cavidades dentárias na investigação de contração de polimerização de resinas compostas (MILCZEWSKI et al., 2006), no interior de 
canais radiculares na avaliação das deformações de cimentos resinosos (KARAM 2015; PULIDO 2016), ex-vivo no ligamento periodontal de animais (KARAM 2012), in vivo no osso mandibular de animais (PEGORINI 2015; KALINOWSKI 2017), etc. Esses sensores permitem a mensuração das deformações e da temperatura possibilitando uma série de estudos na área biológica.

Futuros estudos devem investigar a transferência de temperatura das resinas acrílicas aos ossos cortical e medular em amostras animais ex-vivo recentes. 


\section{CONCLUSÕES}

Considerando-se as limitações do presente estudo pode-se concluir que:

-Não houve diferenças significativas para os valores de temperatura máxima entre a Duralay cervical e apical, GC Pattern LS cervical e apical, Duralay cervical e GC Pattern LS cervical, Duralay apical e GC Pattern LS apical;

-Foram encontradas diferenças para os valores de temperatura máxima entre a Duralay cervical (osso cortical) e a GC Pattern LS apical (osso medular);

-As redes de Bragg apresentaram-se suficientemente sensíveis para a mensuração da variação da transferência de temperatura máxima das resinas acrílicas aos ossos cortical e medular.

Portanto, a partir dos resultados obtidos, podemos afirmar que não há risco de necrose óssea. 


\section{REFERÊNCIAS}

ALBREKTSSON, T.; JOHANSSON, C. Osteoinduction, osteoconduction and osseointegration. European Spine Journal, v. 10, n. 2, p. 96-101. 2001.

ASSIF, D.; NISSAN, J.; VARSANO, I.; SINGER, A. Accuracy of implant impression splinted techniques: Effect of splinting material. International Journal of Oral and Maxillofacial Implants, v. 14, n. 6, p. 885-888. 1999.

BABCHENKO, A.; KHANOKH, B.; SHOMER, Y.; NITZAN, M. Fiber optic sensor for the measurement of the respiratory chest circumference changes. Journal of Biomedical Optics, v. 4, n.2, p. 224-229. 1999.

CABRAL, LM.; GUEDES, CG. Comparative analysis of 4 impression techniques for implants. Implant Dentistry, v. 16, n. 2, p. 187-194. 2007.

CARVALHO, L.; VAZ, M.; SIMÕES, J. Acta of Bioengineering and Biomechanics, v. 4, n. 1, p 801-802. 2003.

CARVALHO, L.; ALBERTO, NJ.; GOME, PS.; NOGUEIRA, RN.; PINTO, JL.; FERNANDES, MH. In the trail of a new bio-sensor for measuring strain in bone: Osteoblastic biocompatibility. Biosensors and Bioelectronic, v. 26, n. 10, p. 4046-4052. 2011.

DENNISON, CR.; WILD, PM.; DVORAK, MF.; WILSON, DR.; CRIPTON, PA. Validation of a novel minimally invasive intervertebral disc pressure sensor utilizing in-fiber Bragg gratings in a porcine model: An ex vivo study. The Spine Journal, v. 33, n. 17, p. 589-594. 2008.

DENNISON, CR.; WILD, PM.; WILSON, DR.; CRIPTON, PA. A minimally invasive in-fiber Bragg grating sensor for intervertebral disc pressure measurements. Measurement Science and Technology, v. 19, n. 8, p. 085201. 2008. 
ERIKKSON, A.; ALBREKTSSON, T.; GRANE, B.; MCQUEEN, D. Thermal injury to bone. A vital-microscopic description of heat effects. International Journal of Oral Surgery, v. 11, n. 2, p. 115-121. 1982.

ERIKSSON, A.; ALBREKTSSON, T. Temperature threshold levels for heatinduced bone tissue injury: A vital-microscopic study in the rabbit. Journal of Prosthetic Dentistry, v. 50, n. 1, p. 101-107. 1983.

FRANCO, APGO.; KARAM, LZ.; GALVÃO, JR.; KALINOWSKI HJ. Analysis of strain and temperature induced by acrylic resins in splinting methods used for dental implant impressions. Journal of Lightwave Technology. (Print), 2016.

FRESVIG, T.; LUDVIGSEN, P.; STEEN, H.; REIKERÅS, O. Fiber optic Bragg grating sensors: An alternative method to strain gauges for measuring deformation in bone. Medical Engineering and Physics, v. 30, n. 1, p. 104108. 2008.

FRIAS, C.; FRAZÃO, O.; TAVARES, S.; VIEIRA, A.; MARQUES, A.; SIMÕES J. Mechanical characterization of bone cement using fiber Bragg grating sensors. Material and Design, v. 30, n. 5, p. 1841-1844. 2009.

GC AMERICA. GC Pattern LS low shrinkage modelling resin tips and tricks: technique and handling of GC Pattern Resin. 2009; 1-12. Disponível em: http://www.gcamerica.com/lab/products/PATTERN RESIN LS/pattern resin te ch guide.pdf. Acesso em: 10 mar. 2017.

GUICHET, DL.; CAPUTO, AA.; CHOI, H.; SORENSEN, JA. Passivity of fit and marginal opening in screw- or cement-retained implant fixed partial denture designs. International Journal Oral Maxillofacial Implants, v. 15, n. 2, p. 239246. 2000.

KALINOWSKI, A.; KARAM, L. Z.; PEGORINI, V.; BIFFE DI RENZO, ANDRE.; SANTOS ROCHA PITTA, CHRISTIANO.; CARDOSO, RAFAEL.; SIMIONI ASSMANN, TANGRIANI.; KALINOWSKI, HYPOLITO.; CARDOZO DA SILVA, JEAN CARLOS. Optical Fiber Bragg Grating Strain Sensor for Bone Stress 
Analysis in Bovine during Masticatory Movements. IEEE Sensors Journal, v. 17, p. 2385-2392, 2017.

KARAM, LEANDRO.; FRANCO, APGO.; PULIDO, CAMILO.; OSNARA, MARIA.; KALINOWSKI, HYPOLITO. In Vitro and In Situ Fiber Bragg Grating Sensor Analysis of Two Dental Resin Cements. Journal of Lightwave Technology, v. 33, p. 2543-2548, 2015.

KARAM, LEANDRO.; MILCZEWSKI, MAURA.; KALINOWSKI, HYPOLITO.; LIAO, YANBIAO.; JIN, WEI.; SAMPSON, DAVID.; YAMAUCHI, RYOZO.; CHUNG, YOUNGJOO.; NAKAMURA, KENTARO.; RAO, YUNJIANG. Strain monitoring of the periodontal ligament in pig's mandibles. In: OFS2012 22nd International Conference on Optical Fiber Sensor, 2012, Beijing. OFS2012 22nd International Conference on Optical Fiber Sensors, 2012. v. 8421.

KARAM, LZ.; FRANCO, APGO.; PULIDO, CA.; GOMES, OMM.; KALINOWSKI, HJ. In Vitro and In Situ Fiber Bragg Grating Sensor Analysis of Two Dental Resin Cements. Journal of Lightwave Technology, v.33, n.12, p.2543-2548. 2015.

KAZEMI, M.; JALALI, H.; EGHTEDARI, M.; SADRIMANESH, R.; SADRESHKEVARI, P.; MAURER, P. Acrylic Resin Polymerization in Direct Contact to the Abutment and the temperature at Bone-Implant Interface: A Pilot In Vidro Study. Journal of Oral Implantology, v. 38, n. 5, p. 595-601. 2012.

MIHAILOV, SJ. Fiber Bragg grating sensors for harsh environments. Sensors, v. 12, n. 2, p. 1898-1918. 2012.

MILCZEWSKI, M.; DA SILVA, J.; ABE, I.; CARVALHO, L.; NOGUEIRA, R.; PATERNO, A.; KALINOWSKI, H.; PINTO, J. Determination of setting expansion of dental materials using fibre optical sensing. Measurement Science and Technology, v. 17, n. 5, p. 1152-1156. 2006. 
ORMIANER, Z.; LAUFER, BZ.; NISSAN, J.; GROSS, M. An investigation of heat transfer to the implant-bone interface related to exothermic heat generation during setting of autopolymerizing acrylic resins applied directly to an implant abutment. International Journal of Oral and Maxillofacial Implants, v. 15, n. 6, p. 837-842. 2000.

OTTEVAERE, H.; $_{\text {; }}$ TABAK, M.; FERNANDEZ, AF.; BERGHMANS, $F_{\text {.; }}$ THIENPONT, H. Optical fiber sensors and their application in monitoring stress build-up in dental resin cements. Proceedings of SPIE, v. 5952, p. 204-216. 2005.

PULIDO, CAMILO ANDRÉS ; DE OLIVEIRA FRANCO, ANA PAULA GEBERT ; GOMES, GIOVANA MONGRUEL ; BITTENCOURT, BRUNA FORTES ; KALINOWSKI, HYPOLITO JOSÉ ; GOMES, João Carlos ; GOMES, Osnara Maria Mongruel. An in situ evaluation of the polymerization shrinkage, degree of conversion, and bond strength of resin cements used for luting fiber posts. The Journal of Prosthetic Dentistry, v. 116, n. 4, p. 570-576, 2016.

RIBEIRO, RICHARDSON; BERTOTTI, FÁBIO; ASSMANN, TANGRIANI. In Vivo Pattern Classification of Ingestive Behavior in Ruminants Using FBG Sensors and Machine Learning. Sensors, v. 15, p. 28456-28471, 2015.

TAKEMAE, R.; UEMURA, T.; OKAMOTO, H.; MATSUI, T.; YOSHIDA, M.; FUKAZAWA, S.; TSUCHIDA, K.; TERUYA, K.; TSUNODA, T. Changes in mental health and quality of life with dental implants as evaluated by General Health Questionnaire (GHQ) and Health Utilities Index (HUI). Environmental Healt and Preventive Medicine, v.17, n. 6, p. 463-473. 2012.

TALAIA, P.; RAMOS, A.; ABE, I.; SCHILLER, M.; LOPES, P.; NOGUEIRA, R.; PINTO, J.; CLARAMUNT, R.; SIMÕES J. Plated and intact femur strains in fracture fixation using fiber Bragg gratings and strain gauges. Experimental Mechanics, v. 47, n. 3, p. 355-363. 2007.

TJIN, S.; TAN, Y.; YOW, M.; LAM, YZ.; HAO, J. Recording compliance of dental splint use in obstructive sleep apnoea patients by force and temperature 
modelling. Medical and Biological Engineering Computing, v. 39, n. 2, p. 182-184. 2001.

VALLITTU PK. Peak temperatures of some prosthetic acrylates on polymerization. Journal of Oral Rehabilitation, v. 23, n.11, p. 504-506. 1996.

VIGOLO, P.; FONZI, F.; MAJZOUB, Z.; CORDIOLI, G. An evaluation of impression techniques for multiple internal connection implant prostheses. Journal of Prosthetic Dentistry, v. 92, n.5, p. 470-476. 2004.

WEHRLE, G.; NOHAMA, P.; KALINOWSKI, HJ.; TORRES, PI.; VALENTE, LCG. A fibre optic Bragg grating strain sensor for monitoring ventilatory movements. Measurement Science and Technology, v.12, n.7, p. 805-809. 2001.

WENNERBERG, A.; ALBREKTSSON T. On implant surfaces: a review of current knowledge and opinions. International Journal of Oral and Maxillofacial Surgery Implants, v.25, n.1, p. 63-74. 2010. 